\title{
On the stochastic nonlinear Schrödinger equations at critical regularities
}

\author{
Tadahiro $\mathrm{Oh}^{1,2}$ (D) Mamoru Okamoto ${ }^{3}$
}

Received: 14 April 2019 / Revised: 20 November 2019 / Published online: 3 January 2020

(c) The Author(s) 2020

\begin{abstract}
We consider the Cauchy problem for the defocusing stochastic nonlinear Schrödinger equations (SNLS) with an additive noise in the mass-critical and energy-critical settings. By adapting the probabilistic perturbation argument employed in the context of the random data Cauchy theory by Bényi et al. (Trans Am Math Soc Ser B 2:1-50, 2015) to the current stochastic PDE setting, we present a concise argument to establish global well-posedness of the mass-critical and energy-critical SNLS.
\end{abstract}

Keywords Stochastic nonlinear Schrödinger equation · Global well-posedness · Mass-critical · Energy-critical · Perturbation theory

Mathematics Subject Classification 35Q55

\section{Introduction}

\subsection{Stochastic nonlinear Schrödinger equations}

We consider the Cauchy problem for the stochastic nonlinear Schrödinger equation (SNLS) with an additive noise:

$\bowtie \quad$ Tadahiro Oh

hiro.oh@ed.ac.uk

Mamoru Okamoto

m_okamoto@shinshu-u.ac.jp

1 School of Mathematics, The University of Edinburgh, James Clerk Maxwell Building, The King's Buildings, Peter Guthrie Tait Road, Edinburgh EH9 3FD, UK

2 The Maxwell Institute for the Mathematical Sciences, James Clerk Maxwell Building, The King's Buildings, Peter Guthrie Tait Road, Edinburgh EH9 3FD, UK

3 Division of Mathematics and Physics, Faculty of Engineering, Shinshu University, 4-17-1 Wakasato, Nagano City 380-8553, Japan 


$$
\left\{\begin{array}{l}
i \partial_{t} u+\Delta u=|u|^{p-1} u+\phi \xi \\
\left.u\right|_{t=0}=u_{0},
\end{array} \quad(t, x) \in \mathbb{R}_{+} \times \mathbb{R}^{d},\right.
$$

where $\xi(t, x)$ denotes a space-time white noise on $\mathbb{R}_{+} \times \mathbb{R}^{d}$ and $\phi$ is a bounded operator on $L^{2}\left(\mathbb{R}^{d}\right)$. In this paper, we restrict our attention to the defocusing case. Our main goal is to present a concise argument in establishing global well-posedness of (1) in the so-called mass-critical and energy-critical cases.

Let us first go over the notion of the scaling-critical regularity for the (deterministic) defocusing nonlinear Schrödinger equation (NLS):

$$
i \partial_{t} u+\Delta u=|u|^{p-1} u,
$$

namely, (1) with $\phi \equiv 0$. The Eq. (2) is known to enjoy the following dilation symmetry:

$$
u(t, x) \longmapsto u^{\lambda}(t, x)=\lambda^{-\frac{2}{p-1}} u\left(\lambda^{-2} t, \lambda^{-1} x\right)
$$

for $\lambda>0$. If $u$ is a solution to (2), then the scaled function $u^{\lambda}$ is also a solution to (2) with the rescaled initial data. This dilation symmetry induces the following scaling-critical Sobolev regularity:

$$
s_{\text {crit }}=\frac{d}{2}-\frac{2}{p-1}
$$

such that the homogeneous $\dot{H}^{s_{\text {crit }}}\left(\mathbb{R}^{d}\right)$-norm is invariant under the dilation symmetry. This critical regularity $s_{\text {crit }}$ provides a threshold regularity for well-posedness and illposedness of (2). Indeed, when $s \geq \max \left(s_{\text {crit }}, 0\right)$, the Cauchy problem (2) is known to be locally well-posed in $H^{s}\left(\mathbb{R}^{d}\right)[6,19,22,36] .{ }^{1}$ On the other hand, it is known that NLS (2) is ill-posed in the scaling supercritical regime: $s<s_{\text {crit }}$. See [9,26,28].

Next, we introduce two important critical regularities associated with the following conservation laws for (2):

$$
\begin{aligned}
\text { Mass: } M(u(t)) & :=\int_{\mathbb{R}^{d}}|u(t, x)|^{2} d x, \\
\text { Energy: } E(u(t)) & :=\frac{1}{2} \int_{\mathbb{R}^{d}}|\nabla u(t, x)|^{2} d x+\frac{d-2}{2 d} \int_{\mathbb{R}^{d}}|u(t, x)|^{\frac{2 d}{d-2}} d x .
\end{aligned}
$$

In view of these conservation laws, we say that the Eq. (2) is

(i) Mass-critical when $s_{\text {crit }}=0$, namely, when $p=1+\frac{4}{d}$,

(ii) Energy-critical when $s_{\text {crit }}=1$, namely, when $p=1+\frac{4}{d-2}$ and $d \geq 3$.

Over the last two decades, we have seen a significant progress in the global-in-time theory of the defocusing NLS (2) in the mass-critical and energy-critical cases [5,11, 15-17,31,34,37]. In particular, we now know that

\footnotetext{
1 When $p$ is not an odd integer, we may need to impose an extra assumption due to the non-smoothness of the nonlinearity. 
(i) The defocusing mass-critical NLS (2) with $p=1+\frac{4}{d}$ is globally well-posed in $L^{2}\left(\mathbb{R}^{d}\right)$

(ii) The defocusing energy-critical NLS (2) with $p=1+\frac{4}{d-2}, d \geq 3$, is globally well-posed in $\dot{H}^{1}\left(\mathbb{R}^{d}\right)$.

Moreover, the following space-time bound on a global solution $u$ to (2) holds:

$$
\|u\|_{L_{t, x}^{\frac{2(d+2)}{d-2 k}}\left(\mathbb{R} \times \mathbb{R}^{d}\right)} \leq C\left(\left\|u_{0}\right\|_{H^{k}}\right)<\infty
$$

with (i) $k=0$ in the mass-critical case and (ii) $k=1$ in the energy-critical case. This bound in particular implies that global-in-time solutions scatter, i.e. they asymptotically behave like linear solutions as $t \rightarrow \pm \infty$.

Let us now turn our attention to SNLS (1). We say that $u$ is a solution to (1) if it satisfies the following Duhamel formulation (= mild formulation):

$$
u(t)=S(t) u_{0}-i \int_{0}^{t} S\left(t-t^{\prime}\right)|u|^{p-1} u\left(t^{\prime}\right) d t^{\prime}-i \int_{0}^{t} S\left(t-t^{\prime}\right) \phi \xi\left(d t^{\prime}\right),
$$

where $S(t)=e^{i t \Delta}$ denotes the linear Schrödinger propagator. The last term on the right-hand side of (4) is called the stochastic convolution, which we denote by $\Psi$. Fix a probability space $(\Omega, \mathcal{F}, P)$ endowed with a filtration $\left\{\mathcal{F}_{t}\right\}_{t \geq 0}$ and let $W$ denote the $L^{2}\left(\mathbb{R}^{d}\right)$-cylindrical Wiener process associated with the filtration $\left\{\mathcal{F}_{t}\right\}_{t \geq 0}$; see (10) below for a precise definition. Then, the stochastic convolution $\Psi$ is defined by

$$
\begin{aligned}
\Psi(t) & =-i \int_{0}^{t} S\left(t-t^{\prime}\right) \phi \xi\left(d t^{\prime}\right) \\
& :=-i \int_{0}^{t} S\left(t-t^{\prime}\right) \phi d W\left(t^{\prime}\right) .
\end{aligned}
$$

See Sect. 2 for the precise meaning of the definition (5); in particular see (11).

Our main goal is to construct global-in-time dynamics for (4) in the mass-critical and energy-critical cases. This means that we take (i) $p=1+\frac{4}{d}$ in the mass-critical case and (ii) $p=1+\frac{4}{d-2}$ in the energy-critical case. Furthermore, we take the stochastic convolution $\Psi$ in (5) to be at the corresponding critical regularity. Suppose that $\phi \in$ $H S\left(L^{2} ; H^{s}\right)$, namely, $\phi$ is a Hilbert-Schmidt operator from $L^{2}\left(\mathbb{R}^{d}\right)$ to $H^{s}\left(\mathbb{R}^{d}\right)$. Then, it is known that $\Psi \in C\left(\mathbb{R}_{+} ; H^{s}\left(\mathbb{R}^{d}\right)\right)$ almost surely; see [12]. Therefore, we will impose that (i) $\phi \in H S\left(L^{2} ; L^{2}\right)$ in the mass-critical case and (ii) $\phi \in H S\left(L^{2} ; H^{1}\right)$ in the energy-critical case.

Previously, de Bouard and Debussche [14] studied SNLS (1) in the energysubcritical setting: $s_{\text {crit }}<1$, assuming that $\phi \in H S\left(L^{2} ; H^{1}\right)$. By using the Strichartz estimates, they showed that the stochastic convolution $\Psi$ almost surely belongs to a right Strichartz space, which allowed them to prove local well-posedness of (1) in $H^{1}\left(\mathbb{R}^{d}\right)$ with $\phi \in H S\left(L^{2} ; H^{1}\right)$ in the energy-subcritical case: $1<p<1+\frac{4}{d-2}$ when $d \geq 3$ and $1<p<\infty$ when $d=1,2$. We point out that when $s \geq \max \left(s_{\text {crit }}, 0\right)$, a slight modification of the argument in [14] with the regularity properties of the 
stochastic convolution (see Lemma 2.2 below) yields local well-posedness ${ }^{2}$ of (1) in $H^{s}\left(\mathbb{R}^{d}\right)$, provided that $\phi \in H S\left(L^{2} ; H^{s}\right)$. See Lemma 2.3 for the statements in the mass-critical and energy-critical cases. We also mention recent papers $[8,30]$ on local well-posedness of (1) with additive noises rougher than the critical regularities, i.e. $\phi \in H S\left(L^{2} ; H^{s}\right)$ with $s<s_{\text {crit }}$.

In the energy-subcritical case, assuming $\phi \in H S\left(L^{2} ; H^{1}\right)$, global well-posedness of (1) in $H^{1}\left(\mathbb{R}^{d}\right)$ follows from an a priori $H^{1}$-bound of solutions to (1) based on the conservation of the energy $E(u)$ for the deterministic NLS and Ito's lemma; see [14]. See also Lemma 2.4. In a recent paper [7], Cheung et al. adapted the $I$-method [10] to the stochastic PDE setting and established global well-posedness of energy-subcritical SNLS below $H^{1}\left(\mathbb{R}^{d}\right)$. In the mass-subcritical case, global well-posedness in $L^{2}\left(\mathbb{R}^{d}\right)$ also follows from an a priori $L^{2}$-bound based on the conservation of the mass $M(u)$ for the deterministic NLS and Ito's lemma.

We extend these global well-posedness results to the mass-critical and energycritical settings.

Theorem 1.1 (i) (Mass-critical case). Let $d \geq 1$ and $p=1+\frac{4}{d}$. Then, given $\phi \in H S\left(L^{2} ; L^{2}\right)$, the defocusing mass-critical SNLS (1) is globally well-posed in $L^{2}\left(\mathbb{R}^{d}\right)$.

(ii) (Energy-critical case). Let $3 \leq d \leq 6$ and $p=1+\frac{4}{d-2}$. Then, given $\phi \in$ $H S\left(L^{2} ; H^{1}\right)$, the defocusing energy-critical SNLS (1) is globally well-posed in $H^{1}\left(\mathbb{R}^{d}\right)$.

In the following, we only consider deterministic initial data $u_{0}$. This assumption is, however, not essential and we may also take random initial data (measurable with respect to the filtration $\mathcal{F}_{0}$ at time 0 ).

In the mass-critical case (and the energy-critical case, respectively), the a priori $L^{2}$-bound (and the a priori $H^{1}$-bound, respectively) does not suffice for global well-posedness (even in the case of the deterministic NLS (2)). The main idea for proving Theorem 1.1 is to adapt the probabilistic perturbation argument introduced by the authors $[4,29]$ in studying global-in-time behavior of solutions to the defocusing energy-critical cubic NLS with random initial data below the energy space. Namely, by letting $v=u-\Psi$, where $\Psi$ is the stochastic convolution defined in (5), we study the equation satisfied by $v$ :

$$
\left\{\begin{array}{l}
i \partial_{t} v+\Delta v=\mathcal{N}(v+\Psi) \\
\left.v\right|_{t=0}=u_{0}
\end{array}\right.
$$

where $\mathcal{N}(u)=|u|^{p-1} u$. Write the nonlinearity as

$$
\mathcal{N}(v+\Psi)=\mathcal{N}(v)+(\mathcal{N}(v+\Psi)-\mathcal{N}(v)) .
$$

Then, the regularity properties of the stochastic convolution (see Lemma 2.2 below) and the fact that their space-time norms can be made small on short time intervals

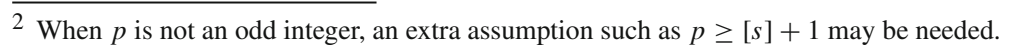


allow us to view the second term on the right-hand side as a perturbative term. By invoking the perturbation lemma (Lemmas 3.2,4.3), we then compare the solution $v$ to (6) with a solution to the deterministic NLS (2) on short time intervals as in [4,29]. See also $[24,35]$ for similar arguments in the deterministic case. In the energy-critical case, we rely on the Lipschitz continuity of $\nabla \mathcal{N}(u)$ in the perturbation argument, which imposes the assumption $d \leq 6$ in Theorem 1.1 .

Remark 1.2 We remark that solutions constructed in this paper are adapted to the given filtration $\left\{\mathcal{F}_{t}\right\}_{t \geq 0}$. For example, adaptedness of a solution $v$ to (6) directly follows from the local-in-time construction of the solution via the Picard iteration. Namely, we consider the map $\Gamma$ defined by

$$
\Gamma v(t):=S(t) u_{0}-i \int_{0}^{t} S\left(t-t^{\prime}\right) \mathcal{N}(v+\Psi)\left(t^{\prime}\right) d t^{\prime} .
$$

Then, we define the $j$ th Picard iterate $P_{j}$ by setting

$$
\begin{aligned}
P_{1} & =S(t) u_{0}, \\
P_{j+1} & =\Gamma P_{j}=S(t) u_{0}-i \int_{0}^{t} S\left(t-t^{\prime}\right) \mathcal{N}\left(P_{j}+\Psi\right)\left(t^{\prime}\right) d t^{\prime}
\end{aligned}
$$

for $j \in \mathbb{N}$. Since the stochastic convolution $\Psi$ is adapted to the filtration $\left\{\mathcal{F}_{t}\right\}_{t \geq 0}$, it is easy to see from (7) that $P_{j}$ is adapted for each $j \in \mathbb{N}$. Furthermore, the local well-posedness of (6) by a contraction mapping principle (see Lemmas 3.1 and 4.1 below) shows that the sequence $\left\{P_{j}\right\}_{j \in \mathbb{N}}$ converges, in appropriate functions spaces, to a limit $v=\lim _{j \rightarrow \infty} P_{j}$, which is a solution to (the mild formulation of) (6). By invoking the closure property of measurability under a limit, we conclude that the solution $v$ to (6) is also adapted to the filtration $\left\{\mathcal{F}_{t}\right\}_{t \geq 0}$. The same comment applies to Lemma 2.3 below.

Remark 1.3 (i) In the focusing case, i.e. with $-|u|^{p-1} u$ in (1), de Bouard and Debussche [13] proved under appropriate conditions that, starting with any initial data, finite-time blowup occurs with positive probability.

(ii) In the mass-subcritical and energy-critical cases, SNLS with a multiplicative noise has been studied in [1-3]. In recent preprints, Fan and Xu [18] and Zhang [39] proved global well-posedness of SNLS with a multiplicative noise in the masscritical and energy-critical setting.

\section{Preliminary results}

In this section, we introduce some notations and go over preliminary results.

Given two separable Hilbert spaces $H$ and $K$, we denote by $H S(H ; K)$ the space of Hilbert-Schmidt operators $\phi$ from $H$ to $K$, endowed with the norm:

$$
\|\phi\|_{H S(H ; K)}=\left(\sum_{n \in \mathbb{N}}\left\|\phi e_{n}\right\|_{K}^{2}\right)^{\frac{1}{2}}
$$


where $\left\{e_{n}\right\}_{n \in \mathbb{N}}$ is an orthonormal basis of $H$.

Since our focus is the mass-critical and energy-critical cases, we introduce $\mathcal{N}_{k}(u)$, $k=0,1$, by

$$
\mathcal{N}_{0}(u):=|u|^{\frac{4}{d}} u \quad \text { and } \quad \mathcal{N}_{1}(u):=|u|^{\frac{4}{d-2}} u .
$$

Namely, $k=0$ corresponds to the mass-critical case, while $k=1$ corresponds to the energy-critical case.

The Strichartz estimates play an important role in our analysis. We say that a pair $(q, r)$ is admissible if $2 \leq q, r \leq \infty,(q, r, d) \neq(2, \infty, 2)$, and

$$
\frac{2}{q}+\frac{d}{r}=\frac{d}{2}
$$

Then, the following Strichartz estimates are known to hold; see [20,23,32,38].

Lemma 2.1 Let $(q, r)$ be admissible. Then, we have

$$
\|S(t) \phi\|_{L_{t}^{q} L_{x}^{r}} \lesssim\|\phi\|_{L^{2}}
$$

For any admissible pair $(\widetilde{q}, \widetilde{r})$, we also have

$$
\left\|\int_{0}^{t} S\left(t-t^{\prime}\right) F\left(t^{\prime}\right) d t^{\prime}\right\|_{L_{t}^{q} L_{x}^{r}} \lesssim\|F\|_{L_{t}^{\widetilde{q}^{\prime}} L_{x}^{\tilde{r}^{\prime}}},
$$

where $\widetilde{q}^{\prime}$ and $\widetilde{r}^{\prime}$ denote the Hölder conjugates. Moreover, if the right-hand side of (9) is finite for some admissible pair $(\widetilde{q}, \widetilde{r})$, then $\int_{0}^{t} S\left(t-t^{\prime}\right) F\left(t^{\prime}\right) d t^{\prime}$ is continuous (in time) with values in $L^{2}\left(\mathbb{R}^{d}\right)$.

Next, we provide a precise meaning to the stochastic convolution defined in (5). Let $(\Omega, \mathcal{F}, P)$ be a probability space endowed with a filtration $\left\{\mathcal{F}_{t}\right\}_{t \geq 0}$. Fix an orthonormal basis $\left\{e_{n}\right\}_{n \in \mathbb{N}}$ of $L^{2}\left(\mathbb{R}^{d}\right)$. We define an $L^{2}\left(\mathbb{R}^{d}\right)$-cylindrical Wiener process $W$ by

$$
W(t, x, \omega):=\sum_{n \in \mathbb{N}} \beta_{n}(t, \omega) e_{n}(x),
$$

where $\left\{\beta_{n}\right\}_{n \in \mathbb{N}}$ is a family of mutually independent complex-valued Brownian motions associated with the filtration $\left\{\mathcal{F}_{t}\right\}_{t \geq 0}$. Here, the complex-valued Brownian motion means that $\operatorname{Re} \beta_{n}(t)$ and $\operatorname{Im} \beta_{n}(t)$ are independent (real-valued) Brownian motions. Then, the space-time white noise $\xi$ is given by a distributional derivative (in time) of $W$ and thus we can express the stochastic convolution $\Psi$ as

$$
\Psi(t)=-i \sum_{n \in \mathbb{N}} \int_{0}^{t} S\left(t-t^{\prime}\right) \phi e_{n} d \beta_{n}\left(t^{\prime}\right),
$$

where each summand is a classical Wiener integral (with respect to the integrator $d \beta_{n}$ ); see [27]. Then, we have the following lemma on the regularity properties of the 
stochastic convolution. See, for example, Proposition 5.9 in [12] for Part (i). As for Part (ii), see [30].

Lemma 2.2 Let $d \geq 1, T>0$, and $s \in \mathbb{R}$. Suppose that $\phi \in H S\left(L^{2} ; H^{s}\right)$.

(i) We have $\Psi \in C\left([0, T] ; H^{s}\left(\mathbb{R}^{d}\right)\right)$ almost surely. Moreover, for any finite $p \geq 1$, there exists $C=C(T, p)>0$ such that

$$
\mathbb{E}\left[\sup _{0 \leq t \leq T}\|\Psi(t)\|_{H^{s}}^{p}\right] \leq C\|\phi\|_{H S\left(L^{2} ; H^{s}\right)}^{p} .
$$

(ii) Given $1 \leq q<\infty$ and finite $r \geq 2$ such that $r \leq \frac{2 d}{d-2}$ when $d \geq 3$, we have $\Psi \in L^{q}\left([0, T] ; W^{s, r}\left(\mathbb{R}^{d}\right)\right)$ almost surely. Moreover, for any finite $p \geq 1$, there exists $C=C(T, p)>0$ such that

$$
\mathbb{E}\left[\|\Psi\|_{L^{q}\left([0, T] ; W^{s, r}\left(\mathbb{R}^{d}\right)\right)}^{p}\right] \leq C\|\phi\|_{H S\left(L^{2} ; H^{s}\right)}^{p} .
$$

By the Strichartz estimates (Lemma 2.1) and Lemma 2.2 on the stochastic convolution, one can easily prove the following local well-posedness (see Lemma 2.3 below) of the mass-critical and energy-critical SNLS (1) by essentially following the argument in [14], namely, by studying the Duhamel formulation for $v=u-\Psi$ :

$$
v(t)=S(t) u_{0}-i \int_{0}^{t} S\left(t-t^{\prime}\right) \mathcal{N}(v+\Psi)\left(t^{\prime}\right) d t^{\prime} .
$$

See also Lemmas 3.1 and 4.1 below. In the mass-critical case, the admissible pair $q=r=\frac{2(d+2)}{d}$ plays an important role. In the energy-critical case, we use the following admissible pair

$$
\left(q_{d}, r_{d}\right):=\left(\frac{2 d}{d-2}, \frac{2 d^{2}}{d^{2}-2 d+4}\right)
$$

for $d \geq 3$.

Lemma 2.3 (i) (Mass-critical case). Let $d \geq 1, p=1+\frac{4}{d}$, and $\phi \in H S\left(L^{2} ; L^{2}\right)$. Then, given any $u_{0} \in L^{2}\left(\mathbb{R}^{d}\right)$, there exists an almost surely positive stopping time $T=T_{\omega}\left(u_{0}\right)$ and a unique local-in-time solution $u \in C\left([0, T] ; L^{2}\left(\mathbb{R}^{d}\right)\right)$ to the mass-critical SNLS (1). Furthermore, the following blowup alternative holds; let $T^{*}=T_{\omega}^{*}\left(u_{0}\right)$ be the forward maximal time of existence. Then, either

$$
T^{*}=\infty \quad \text { or } \quad \lim _{T \nearrow T^{*}}\|u\|_{L_{t, x}^{(d+2)}}^{\frac{\left.2(d+2) \times \mathbb{R}^{d}\right)}{([0, T)}}=\infty .
$$

(ii) (Energy-critical case). Let $3 \leq d \leq 6, p=1+\frac{4}{d-2}$, and $\phi \in H S\left(L^{2} ; H^{1}\right)$. Then, given any $u_{0} \in H^{1}\left(\mathbb{R}^{d}\right)$, there exists an almost surely positive stopping time $T=T_{\omega}\left(u_{0}\right)$ and a unique local-in-time solution $u \in C\left([0, T] ; H^{1}\left(\mathbb{R}^{d}\right)\right)$ to the 
energy-critical SNLS (1). Furthermore, the following blowup alternative holds; let $T^{*}=T_{\omega}^{*}\left(u_{0}\right)$ be the forward maximal time of existence. Then, either

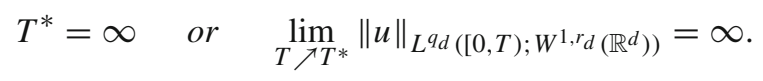

We note that the mapping: $\left(u_{0}, \Psi\right) \mapsto v$ is continuous. See Proposition 3.5 in [14]. In the energy-critical case, the local-in-time well-posedness also holds for $d>6$ (see Remark 4.2 below). As mentioned earlier, the perturbation argument requires the Lipschitz continuity of $\nabla \mathcal{N}$ and hence we need to assume $d \leq 6$ in the following.

Lastly, we state the a priori bounds on the mass and energy of solutions constructed in Lemma 2.3.

Lemma 2.4 (i) (Mass-critical case). Assume the hypotheses in Lemma 2.3(i). Then, given $T_{0}>0$, there exists $C_{1}=C_{1}\left(M\left(u_{0}\right), T_{0},\|\phi\|_{H S\left(L^{2} ; L^{2}\right)}\right)>0$ such that for any stopping time $T$ with $0<T<\min \left(T^{*}, T_{0}\right)$ almost surely, we have

$$
\mathbb{E}\left[\sup _{0 \leq t \leq T} M(u(t))\right] \leq C_{1}
$$

where $u$ is the solution to the mass-critical SNLS (1) with $\left.u\right|_{t=0}=u_{0}$ and $T^{*}=$ $T_{\omega}^{*}\left(u_{0}\right)$ is the forward maximal time of existence.

(ii) (Energy-critical case). Assume the hypotheses in Lemma 2.3(ii). Then, given $T_{0}>$ 0 , there exists $C_{2}=C_{2}\left(M\left(u_{0}\right), E\left(u_{0}\right), T_{0},\|\phi\|_{H S\left(L^{2} ; H^{1}\right)}\right)>0$ such that for any stopping time $T$ with $0<T<\min \left(T^{*}, T_{0}\right)$ almost surely, we have

$$
\mathbb{E}\left[\sup _{0 \leq t \leq T} M(u(t))\right]+\mathbb{E}\left[\sup _{0 \leq t \leq T} E(u(t))\right] \leq C_{2},
$$

where $u$ is the solution to the defocusing energy-critical SNLS (1) with $\left.u\right|_{t=0}=u_{0}$ and $T^{*}=T_{\omega}^{*}\left(u_{0}\right)$ is the forward maximal time of existence.

For Part (ii), we need to assume that the equation is defocusing. These a priori bounds follow from Ito's lemma and the Burkholder-Davis-Gundy inequality. In order to justify an application of Ito's lemma, one needs to go through a certain approximation argument. See, for example, Proposition 3.2 in [14]. In our masscritical and energy-critical settings, however, such an approximation argument is more involved and hence we present a sketch of the argument in "Appendix A".

\section{Mass-critical case}

In this section, we prove global well-posedness of the defocusing mass-critical SNLS (1) (Theorem 1.1(i)). In Sect. 3.1, we first study the following defocusing mass-critical NLS with a deterministic perturbation:

$$
i \partial_{t} v+\Delta v=\mathcal{N}_{0}(v+f),
$$


where $\mathcal{N}_{0}$ is as in (8) and $f$ is a given deterministic function, satisfying certain regularity conditions. By applying the perturbation lemma, we prove global existence for (14), assuming an a priori $L^{2}$-bound of a solution $v$ to (14). See Proposition 3.3. In Sect. 3.2, we then present the proof of Theorem 1.1(i) by writing (1) in the form (14) (with $f=\Psi$ ) and verifying the hypotheses in Proposition 3.3.

\subsection{Mass-critical NLS with a perturbation}

By the standard Strichartz theory, we have the following local well-posedness of the perturbed NLS (14).

Lemma 3.1 There exists small $\eta_{0}>0$ such that if

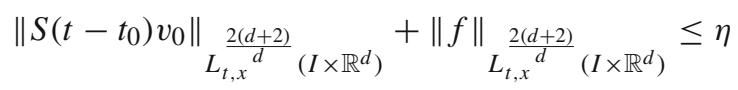

for some $\eta \leq \eta_{0}$ and some time interval $I=\left[t_{0}, t_{1}\right] \subset \mathbb{R}$, then there exists a unique solution $v \in C\left(I ; L^{2}\left(\mathbb{R}^{d}\right)\right) \cap L_{t, x}^{\frac{2(d+2)}{d}}\left(I \times \mathbb{R}^{d}\right)$ to $(14)$ with $v\left(t_{0}\right)=v_{0} \in L^{2}\left(\mathbb{R}^{d}\right)$. Moreover, we have

$$
\|v\| \underset{L_{t, x}}{\frac{2(d+2)}{d}\left(I \times \mathbb{R}^{d}\right)} \leq 2 \eta
$$

Proof We show that the map $\Gamma$ defined by

$$
\Gamma v(t):=S\left(t-t_{0}\right) v_{0}-i \int_{t_{0}}^{t} S\left(t-t^{\prime}\right) \mathcal{N}_{0}(v+f)\left(t^{\prime}\right) d t^{\prime}
$$

is a contraction on the ball $B_{2 \eta} \subset L_{t, x}^{\frac{2(d+2)}{d}}\left(I \times \mathbb{R}^{d}\right)$ of radius $2 \eta>0$ centered at the origin, provided that $\eta>0$ is sufficiently small. Noting that the Hölder conjugate of $\frac{2(d+2)}{d}$ is $\frac{2(d+2)}{d+4}=\frac{2(d+2)}{d} /\left(1+\frac{4}{d}\right)$, it follows from Lemma 2.1 that there exists small $\eta_{0}>0$ such that

$$
\begin{aligned}
& \|\Gamma v\|_{L_{t, x}^{\frac{2(d+2)}{d}}\left(I \times \mathbb{R}^{d}\right)} \leq\left\|S\left(t-t_{0}\right) v_{0}\right\|_{L_{t, x}^{\frac{2(d+2)}{d}}\left(I \times \mathbb{R}^{d}\right)}+\left\|\Gamma v-S\left(t-t_{0}\right) v_{0}\right\|_{L_{t, x}^{\frac{2(d+2)}{d}}}\left(I \times \mathbb{R}^{d}\right)
\end{aligned}
$$

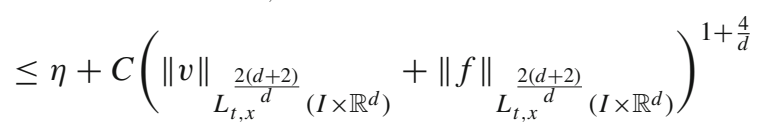

$$
\begin{aligned}
& \leq \eta+C \eta^{1+\frac{4}{d}} \leq 2 \eta
\end{aligned}
$$

and

$$
\left\|\Gamma v_{1}-\Gamma v_{2}\right\| \underset{L_{t, x}{ }^{\frac{2(d+2)}{d}}}{\left(I \times \mathbb{R}^{d}\right)} \leq \leq \frac{1}{2}\left\|v_{1}-v_{2}\right\| \underset{L_{t, x} \frac{2(d+2)}{d}}{\left(I \times \mathbb{R}^{d}\right)}
$$


for any $v, v_{1}, v_{2} \in B_{2 \eta}$ and $0<\eta \leq \eta_{0}$. Hence, $\Gamma$ is a contraction on $B_{2 \eta}$. Furthermore, we have

$$
\begin{aligned}
\|v\|_{L^{\infty}\left(I ; L^{2}\left(\mathbb{R}^{d}\right)\right) \leq} & \left\|S\left(t-t_{0}\right) v_{0}\right\|_{L^{\infty}\left(I ; L^{2}\left(\mathbb{R}^{d}\right)\right)} \\
& +C\left(\|v\| \underset{L_{t, x}^{\frac{2(d+2)}{d}}}{\left(I \times \mathbb{R}^{d}\right)}+\|f\|_{L_{t, x}}^{\frac{2(d+2)}{d}} \underset{\left(I \times \mathbb{R}^{d}\right)}{ }\right)^{1+\frac{4}{d}} \\
\leq & \left\|v_{0}\right\|_{L^{2}}+C \eta^{1+\frac{4}{d}}<\infty
\end{aligned}
$$

for any $v \in B_{2 \eta}$. This shows that $v \in C\left(I ; L^{2}\left(\mathbb{R}^{d}\right)\right)$.

Next, we recall the long-time stability result in the mass-critical setting. See [35] for the proof.

Lemma 3.2 (Mass-critical perturbation lemma) Let I be a compact interval. Suppose that $v \in C\left(I ; L^{2}\left(\mathbb{R}^{d}\right)\right)$ satisfies the following perturbed $N L S$ :

$$
i \partial_{t} v+\Delta v=|v|^{\frac{4}{d}} v+e
$$

satisfying

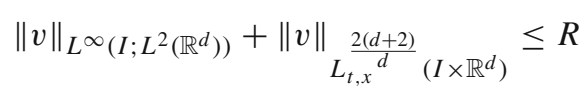

for some $R \geq 1$. Then, there exists $\varepsilon_{0}=\varepsilon_{0}(R)>0$ such that if we have

$$
\left\|u_{0}-v\left(t_{0}\right)\right\|_{L^{2}\left(\mathbb{R}^{d}\right)}+\|e\|_{L_{t, x}^{\frac{2(d+2)}{d+4}}\left(I \times \mathbb{R}^{d}\right)} \leq \varepsilon
$$

for some $u_{0} \in L^{2}\left(\mathbb{R}^{d}\right)$, some $t_{0} \in I$, and some $\varepsilon<\varepsilon_{0}$, then there exists a solution $u \in C\left(I ; L^{2}\left(\mathbb{R}^{d}\right)\right)$ to the defocusing mass-critical NLS:

$$
i \partial_{t} u+\Delta u=|u|^{\frac{4}{d}} u
$$

with $u\left(t_{0}\right)=u_{0}$ such that

$$
\begin{aligned}
\|u\|_{L^{\infty}\left(I ; L^{2}\left(\mathbb{R}^{d}\right)\right)}+\|u\|{ }_{L_{t, x}^{\frac{2(d+2)}{d}}\left(I \times \mathbb{R}^{d}\right)} & \leq C_{1}(R), \\
\|u-v\|_{L^{\infty}\left(I ; L^{2}\left(\mathbb{R}^{d}\right)\right)}+\|u-v\| \underbrace{}_{L_{t, x}^{\frac{2(d+2)}{d}}}\left(I \times \mathbb{R}^{d}\right) & \leq C_{1}(R) \varepsilon,
\end{aligned}
$$

where $C_{1}(R)$ is a non-decreasing function of $R$.

In the remaining part of this subsection, we consider long time existence of solutions to the perturbed NLS (14) under several assumptions. Given $T>0$, we assume that there exist $C, \theta>0$ such that

$$
\|f\|_{L_{t, x} \frac{2(d+2)}{d}\left(I \times \mathbb{R}^{d}\right)} \leq C|I|^{\theta}
$$


for any interval $I \subset[0, T]$. Then, Lemma 3.1 guarantees existence of a solution to the perturbed NLS (14), at least for a short time. The following proposition establishes long time existence under some hypotheses.

Proposition 3.3 Given $T>0$, assume the following conditions (i)-(ii):

(i) $f \in L_{t, x}^{\frac{2(d+2)}{d}}\left([0, T] \times \mathbb{R}^{d}\right)$ satisfies (18),

(ii) Given a solution $v$ to (14), the following a priori $L^{2}$-bound holds:

$$
\|v\|_{L^{\infty}\left([0, T] ; L^{2}\left(\mathbb{R}^{d}\right)\right)} \leq R
$$

for some $R \geq 1$.

Then, there exists $\tau=\tau(R, \theta)>0$ such that, given any $t_{0} \in[0, T)$, a unique solution $v$ to (14) exists on $\left[t_{0}, t_{0}+\tau\right] \cap[0, T]$. In particular, the condition (ii) guarantees existence of a unique solution $v$ to the perturbed $N L S$ (14) on the entire interval $[0, T]$.

Proof By setting $e=\mathcal{N}_{0}(v+f)-\mathcal{N}_{0}(v)$, Eq. (14) reduces to (15). In the following, we iteratively apply Lemma 3.2 on short intervals and show that there exists $\tau=$ $\tau(R, \theta)>0$ such that (15) is well-posed on $\left[t_{0}, t_{0}+\tau\right] \cap[0, T]$ for any $t_{0} \in[0, T)$.

Let $w$ be the global solution to the defocusing mass-critical NLS (17) with $w\left(t_{0}\right)=$ $v\left(t_{0}\right)=v_{0}$. By the assumption (19), we have $\left\|w\left(t_{0}\right)\right\|_{L^{2}} \leq R$. Then, by the space-time bound (3), we have

$$
\|w\|_{L_{t, x}^{\frac{2(d+2)}{d}}\left(\mathbb{R} \times \mathbb{R}^{d}\right)} \leq C(R)<\infty .
$$

Given small $\eta>0$ (to be chosen later), we divide the interval $\left[t_{0}, T\right]$ into $J=$ $J(R, \eta) \sim(C(R) / \eta)^{\frac{2(d+2)}{d}}$ many subintervals $I_{j}=\left[t_{j}, t_{j+1}\right]$ such that

$$
\|w\| \underset{L_{t, x}{ }^{\frac{2(d+2)}{d}}\left(I_{j} \times \mathbb{R}^{d}\right)}{ } \leq \eta
$$

We point out that $\eta$ will be chosen as an absolute constant and hence dependence of other constants on $\eta$ is not essential in the following. Fix $\tau>0$ (to be chosen later in terms of $R$ and $\theta)$ and write $\left[t_{0}, t_{0}+\tau\right]=\bigcup_{j=0}^{J^{\prime}}\left(\left[t_{0}, t_{0}+\tau\right] \cap I_{j}\right)$ for some $J^{\prime} \leq J-1$, where $\left[t_{0}, t_{0}+\tau\right] \cap I_{j} \neq \emptyset$ for $0 \leq j \leq J^{\prime}$ and $\left[t_{0}, t_{0}+\tau\right] \cap I_{j}=\emptyset$ for $j>J^{\prime}$.

Since the nonlinear evolution $w$ is small on each $I_{j}$, it follows that the linear evolution $S\left(t-t_{j}\right) w\left(t_{j}\right)$ is also small on each $I_{j}$. Indeed, from the Duhamel formula, we have

$$
S\left(t-t_{j}\right) w\left(t_{j}\right)=w(t)-i \int_{t_{j}}^{t} S\left(t-t^{\prime}\right) \mathcal{N}_{0}(w)\left(t^{\prime}\right) d t^{\prime}
$$


Then, by Lemma 2.1 and (20), we have

$$
\begin{aligned}
\left\|S\left(t-t_{j}\right) w\left(t_{j}\right)\right\|_{L_{t, x}^{\frac{2(d+2)}{d}}}^{\frac{2\left(I_{j} \times \mathbb{R}^{d}\right)}{}} & \leq\|w\|{ }_{L_{t, x}^{\frac{2(d+2)}{d}}} \\
& \leq \eta+C \eta^{1+\frac{4}{d}} \\
& \leq 2 \eta
\end{aligned}
$$

for all $j=0, \ldots, J-1$, provided that $\eta>0$ is sufficiently small.

Now, we estimate $v$ on the first interval $I_{0}$. By $v\left(t_{0}\right)=w\left(t_{0}\right)$ and (21), we have

$$
\left\|S\left(t-t_{0}\right) v\left(t_{0}\right)\right\|_{L_{t, x}^{\frac{2(d+2)}{d}}}^{\frac{\left(I_{0} \times \mathbb{R}^{d}\right)}{}}=\left\|S\left(t-t_{0}\right) w\left(t_{0}\right)\right\|_{L_{t, x}^{\frac{2(d+2)}{d}}\left(I_{0} \times \mathbb{R}^{d}\right)} \leq 2 \eta .
$$

Let $\eta_{0}>0$ be as in Lemma 3.1. Then, by the local theory (Lemma 3.1), we have

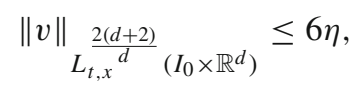

as long as $3 \eta<\eta_{0}$ and $\tau=\tau(\eta, \theta)=\tau(\theta)>0$ is sufficiently small so that

$$
\|f\|_{L_{t, x}^{\frac{2(d+2)}{d}}\left(\left[t_{0}, t_{0}+\tau\right)\right)} \leq C \tau^{\theta} \leq \eta .
$$

Next, we estimate the error term. By Lemma 2.1 and (18), we have

$$
\begin{aligned}
\|e\|_{L_{t, x}^{\frac{2(d+2)}{d+4}}\left(I_{0} \times \mathbb{R}^{d}\right)} & \leq C\left(\|v\|_{L_{t, x}^{\frac{2(d+2)}{d}}}\left(I_{0} \times \mathbb{R}^{d}\right)\right. \\
& \leq C\left(\eta+\tau^{\theta}\right)^{\frac{4}{d}} \tau^{\theta} \\
& \leq C \tau^{\theta}
\end{aligned}
$$

for any small $\eta, \tau>0$. Given $\varepsilon>0$, we can choose $\tau=\tau(\varepsilon, \theta)>0$ sufficiently small so that

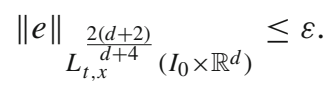

In particular, for $\varepsilon<\varepsilon_{0}$ with $\varepsilon_{0}=\varepsilon_{0}(R)>0$ dictated by Lemma 3.2, the condition (16) is satisfied on $I_{0}$. Hence, by the perturbation lemma (Lemma 3.2), we obtain

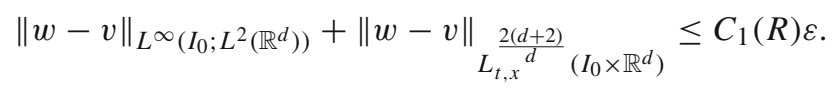

In particular, we have

$$
\left\|w\left(t_{1}\right)-v\left(t_{1}\right)\right\|_{L^{2}\left(\mathbb{R}^{d}\right)} \leq C_{1}(R) \varepsilon .
$$


We now move onto the second interval $I_{1}$. By (21) and Lemma 2.1 with (24), we have

$$
\begin{aligned}
& \left\|S\left(t-t_{1}\right) v\left(t_{1}\right)\right\|{ }_{L_{t, x}}^{\frac{2(d+2)}{d}}\left(I_{1} \times \mathbb{R}^{d}\right) \\
& \quad \leq\left\|S\left(t-t_{1}\right) w\left(t_{1}\right)\right\|{ }_{L_{t, x}^{\frac{2(d+2)}{d}}}\left(I_{1} \times \mathbb{R}^{d}\right) \\
& \quad \leq 2 \eta+C_{0} \cdot C_{1}(R) \varepsilon \leq 3 \eta
\end{aligned}
$$

by choosing $\varepsilon=\varepsilon(R, \eta)=\varepsilon(R)>0$ sufficiently small.

Proceeding as before, it follows from Lemma 3.1 with (25) that

$$
\|v\| \underset{L_{t, x}^{\frac{2(d+2)}{d}}\left(I_{1} \times \mathbb{R}^{d}\right)}{ } \leq 8 \eta
$$

as long as $4 \eta \leq \eta_{0}$ and $\tau>0$ is sufficiently small so that (22) is satisfied. By repeating the computation in (23) with (18), we have

$$
\|e\|_{L_{t, x}^{\frac{2(d+2)}{d+4}}\left(I_{1} \times \mathbb{R}^{d}\right)} \leq C \tau^{\theta} \leq \varepsilon
$$

by choosing $\tau=\tau(\varepsilon, \theta)>0$ sufficiently small. Hence, by the perturbation lemma (Lemma 3.2) applied to the second interval $I_{1}$, we obtain

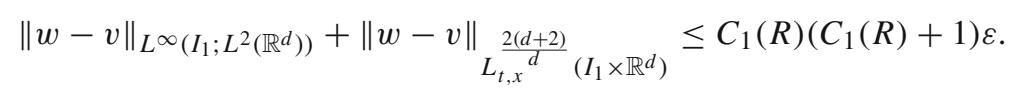

provided that $\tau=\tau(\varepsilon, \theta)>0$ is chosen sufficiently small and that $\left(C_{1}(R)+1\right) \varepsilon<\varepsilon_{0}$. In particular, we have

$$
\left\|w\left(t_{2}\right)-v\left(t_{2}\right)\right\|_{L^{2}\left(\mathbb{R}^{d}\right)} \leq C_{1}(R)\left(C_{1}(R)+1\right) \varepsilon=: C_{2}(R) \varepsilon
$$

For $j \geq 2$, define $C_{j}(R)$ recursively by setting

$$
C_{j}(R)=C_{1}(R)\left(C_{j-1}(R)+1\right)
$$

Then, proceeding inductively, we obtain

$$
\left\|w\left(t_{j}\right)-v\left(t_{j}\right)\right\|_{L^{2}\left(\mathbb{R}^{d}\right)} \leq C_{j}(R) \varepsilon,
$$

for all $0 \leq j \leq J^{\prime}$, as long as $\varepsilon=\varepsilon(R, \eta, J)>0$ is sufficiently small such that

- $C_{0} \cdot C_{j}(R) \varepsilon \leq \eta$ (here, $C_{0}$ is the constant from the Strichartz estimate in (25)),

- $\left(C_{j}(R)+1\right) \varepsilon<\varepsilon_{0}$,

for $j=1, \ldots, J^{\prime}$. Recalling that $J^{\prime}+1 \leq J=J(R, \eta)$, we see that this can be achieved by choosing small $\eta>0, \varepsilon=\varepsilon(R, \eta)=\varepsilon(R)>0$, and $\tau=\tau(\varepsilon, \theta)=$ $\tau(R, \theta)>0$ sufficiently small. This guarantees existence of a (unique) solution $v$ to (14) on $\left[t_{0}, t_{0}+\tau\right]$. Lastly, noting that $\tau>0$ is independent of $t_{0} \in[0, T)$, we conclude existence of the solution $v$ to $(14)$ on the entire interval $[0, T]$. 


\subsection{Proof of Theorem 1.1(i)}

We are now ready to present a proof of Theorem 1.1(i). Given a local-in-time solution $u$ to (1), let $v=u-\Psi$. Then, $v$ satisfies

$$
\left\{\begin{array}{l}
i \partial_{t} v+\Delta v=\mathcal{N}_{0}(v+\Psi) \\
\left.v\right|_{t=0}=u_{0}
\end{array}\right.
$$

Theorem 1.1(i) follows from applying Proposition 3.3 to (26) with $f=\Psi$, once we verify the hypotheses (i) and (ii).

Fix $T>0$. From Lemma 2.4 and Markov's inequality, we have the following almost sure a priori bound:

$$
\sup _{0 \leq t \leq T} M(u(t)) \leq C\left(\omega, T, M\left(u_{0}\right),\|\phi\|_{H S\left(L^{2} ; L^{2}\right)}\right)<\infty
$$

for a solution $u$ to (1) with $p=1+\frac{4}{d}$. Then, from (27) and Lemma 2.2(i), we obtain

$$
\begin{aligned}
\sup _{0 \leq t \leq T} M(v(t)) & =\sup _{0 \leq t \leq T} M(u(t)-\Psi(t)) \leq \sup _{0 \leq t \leq T} M(u(t))+\sup _{0 \leq t \leq T} M(\Psi(t)) \\
& \leq C\left(\omega, T, M\left(u_{0}\right),\|\phi\|_{H S\left(L^{2} ; L^{2}\right)}\right)<\infty
\end{aligned}
$$

almost surely. This shows that the hypothesis (ii) in Proposition 3.3 holds almost surely for some almost surely finite $R=R(\omega) \geq 1$. The hypothesis (i) in Proposition 3.3 easily follows from Hölder's inequality in time, Markov's inequality, and Lemma 2.2(ii). More precisely, by fixing finite $q>\frac{2(d+2)}{d}$ and noting $\frac{2(d+2)}{d} \leq \frac{2 d}{d-2}$ for $d \geq 3$, Lemma 2.2(ii) yields

$$
\mathbb{E}\left[\|\Psi\|_{L^{q}\left([0, T] ; L^{\frac{2(d+2)}{d}}\left(\mathbb{R}^{d}\right)\right)}\right] \leq C\|\phi\|_{H S\left(L^{2} ; L^{2}\right)} .
$$

Then, Markov's inequality yields

$$
\|\Psi\|_{L^{q}\left([0, T] ; L^{\frac{2(d+2)}{d}}\left(\mathbb{R}^{d}\right)\right)} \leq C\left(\omega,\|\phi\|_{H S\left(L^{2} ; L^{2}\right)}\right)<\infty,
$$

which in turn implies $\Psi \in L_{t, x}^{\frac{2(d+2)}{d}}\left([0, T] \times \mathbb{R}^{d}\right)$ almost surely. Moreover, it follows from (28) and Hölder's inequality in time that

$$
\|\Psi\|_{L_{t, x}^{\frac{2(d+2)}{d}}\left(I \times \mathbb{R}^{d}\right)} \leq|I|^{\theta}\|\Psi\|_{L^{q}\left(I ; L^{\frac{2(d+2)}{d}}\left(\mathbb{R}^{d}\right)\right)} \leq C\left(\omega,\|\phi\|_{H S\left(L^{2} ; L^{2}\right)}\right)|I|^{\theta}
$$

for any interval $I \subset[0, T]$, where $\theta=\frac{d}{2(d+2)}-\frac{1}{q}>0$. This verifies (18).

Hence, by applying Proposition 3.3, we can construct a solution $v$ to $(26)$ on $[0, T]$. Since the choice of $T>0$ was arbitrary, this proves Theorem 1.1(i). 


\section{Energy-critical case}

In this section, we prove global well-posedness of the defocusing energy-critical SNLS (1) (Theorem 1.1(ii)). The idea is to follow the argument for the mass-critical case presented in Sect. 3. Namely, we study the following defocusing energy-critical NLS with a deterministic perturbation:

$$
i \partial_{t} v+\Delta v=\mathcal{N}_{1}(v+f)
$$

where $\mathcal{N}_{1}$ is as in (8) and $f$ is a given deterministic function, satisfying certain regularity conditions.

Let $q_{d}$ and $r_{d}$ be as in (12) and set $\rho_{d}:=\frac{2 d^{2}}{(d-2)^{2}}$ for $d \geq 3$. A direct calculation shows that

$$
\frac{d+2}{d-2} \frac{1}{q_{d}}=\frac{1}{q_{d}^{\prime}}, \quad \frac{1}{r_{d}^{\prime}}=\frac{1}{r_{d}}+\frac{4}{d-2} \frac{1}{\rho_{d}}, \quad \text { and } \quad W^{1, r_{d}}\left(\mathbb{R}^{d}\right) \hookrightarrow L^{\rho_{d}}\left(\mathbb{R}^{d}\right) .
$$

\subsection{Energy-critical NLS with a perturbation}

We first go over the local theory for the perturbed NLS (29) in the energy-critical case.

Lemma 4.1 Let $3 \leq d \leq 6$. Then, there exists small $\eta_{0}=\eta_{0}>0$ such that if

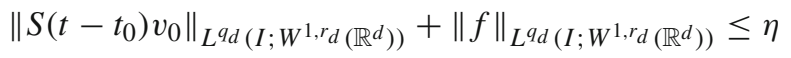

for some $\eta \leq \eta_{0}$ and some time interval $I=\left[t_{0}, t_{1}\right] \subset \mathbb{R}$, then there exists a unique solution $v \in C\left(I ; H^{1}\left(\mathbb{R}^{d}\right)\right) \cap L^{q_{d}}\left(I ; W^{1, r_{d}}\left(\mathbb{R}^{d}\right)\right)$ to $(29)$ with $v\left(t_{0}\right)=v_{0} \in H^{1}\left(\mathbb{R}^{d}\right)$. Moreover, we have

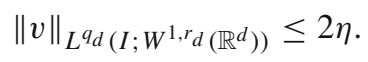

Proof We show that the map $\Gamma$ defined by

$$
\Gamma v(t):=S\left(t-t_{0}\right) v_{0}-i \int_{t_{0}}^{t} S\left(t-t^{\prime}\right) \mathcal{N}_{1}(v+f)\left(t^{\prime}\right) d t^{\prime}
$$

is a contraction on $B_{2 \eta} \subset L^{q_{d}}\left(I ; W^{1, r_{d}}\left(\mathbb{R}^{d}\right)\right)$ of radius $2 \eta>0$ centered at the origin, provided that $\eta>0$ is sufficiently small. It follows from Lemma 2.1 and (30) with (31) that there exists small $\eta_{0}>0$ such that

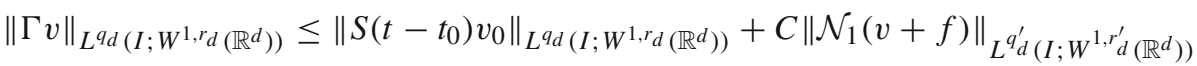

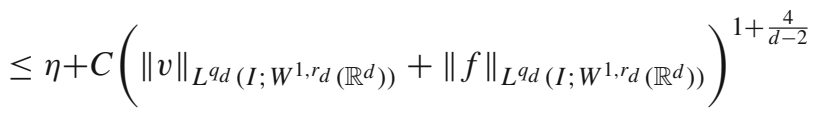

$$
\begin{aligned}
& \leq \eta+C \eta^{1+\frac{4}{d-2}} \leq 2 \eta
\end{aligned}
$$


for $v \in B_{2 \eta}$ and $0<\eta \leq \eta_{0}$. Recall that $\nabla \mathcal{N}_{1}$ is Lipschitz continuous when $3 \leq d \leq 6$ and we have

$$
\begin{aligned}
\nabla N_{1}\left(u_{1}\right)-\nabla \mathcal{N}_{1}\left(u_{2}\right) & =O\left(\left|u_{1}\right|^{\frac{4}{d-2}}+\left|u_{1}\right|^{\frac{4}{d-2}}\right) \nabla\left(u_{1}-u_{2}\right) \\
& +O\left(\left(\left|u_{1}\right|^{\frac{6-d}{d-2}}+\left|u_{1}\right|^{\frac{6-d}{d-2}}\right)\left|u_{1}-u_{2}\right|\right) \nabla u_{2} .
\end{aligned}
$$

See, for example, Case 4 in the proof of Proposition 4.1 in [29]. Then, proceeding as above with (32), we have

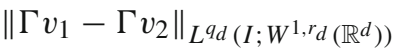

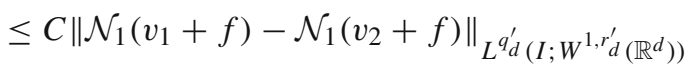

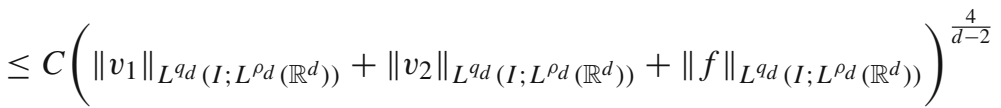

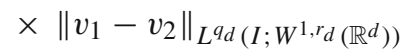

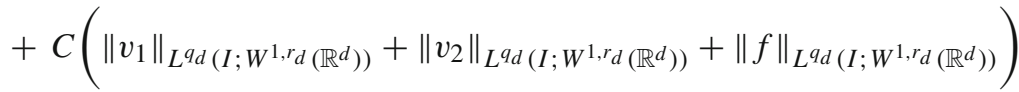

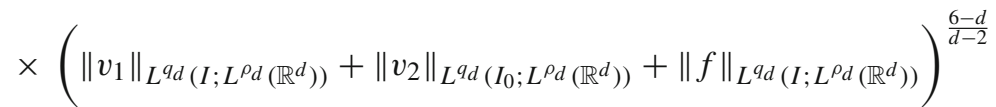

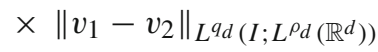

$$
\begin{aligned}
& \leq C \eta^{\frac{4}{d-2}}\left\|v_{1}-v_{2}\right\|_{\left.L^{q_{d}\left(I ; W^{1, r}\right.} d\left(\mathbb{R}^{d}\right)\right)}
\end{aligned}
$$

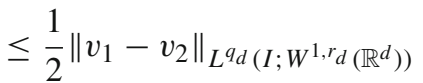

for $v_{1}, v_{2} \in B_{2 \eta}$ and $0<\eta \leq \eta_{0}$. Hence, $\Gamma$ is a contraction on $B_{2 \eta}$. Furthermore, we have

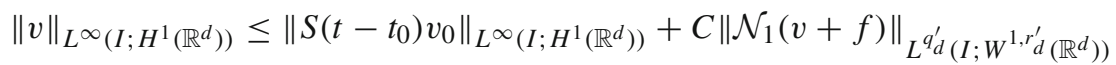

$$
\begin{aligned}
& \leq\left\|v_{0}\right\|_{H^{1}}+C \eta^{1+\frac{4}{d-2}}<\infty
\end{aligned}
$$

for $v \in B_{2 \eta}$. This shows that $v \in C\left(I ; H^{1}\left(\mathbb{R}^{d}\right)\right)$.

Remark 4.2 The restriction $d \leq 6$ appears in (32) and (33), where we used the Lipschitz continuity of $\nabla \mathcal{N}_{1}$. Following the argument in [6], we can remove this restriction and construct a solution by carrying out a contraction argument on $B_{2 \eta} \subset L^{q_{d}}\left(I ; W^{1, r_{d}}\left(\mathbb{R}^{d}\right)\right)$ equipped with the distance

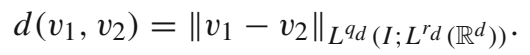

Indeed, a slight modification of the computation in (33) shows $d\left(\Gamma v_{1}, \Gamma v_{2}\right) \leq$ $\frac{1}{2} d\left(v_{1}, v_{2}\right)$ for any $v_{1}, v_{2} \in B_{2 \eta}$. 
Next, we state the long-time stability result in the energy-critical setting. See [11, $25,33,35]$. The following lemma is stated in terms of non-homogeneous spaces, the proof follows closely to that in the mass-critical case.

Lemma 4.3 (Energy-critical perturbation lemma) Let $3 \leq d \leq 6$ and I be a compact interval. Suppose that $v \in C\left(I ; H^{1}\left(\mathbb{R}^{d}\right)\right)$ satisfies the following perturbed NLS:

$$
i \partial_{t} v+\Delta v=|v|^{\frac{4}{d-2}} v+e
$$

satisfying

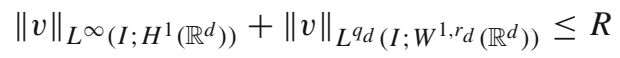

for some $R \geq 1$. Then, there exists $\varepsilon_{0}=\varepsilon_{0}(R)>0$ such that if we have

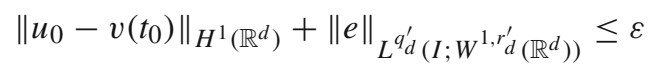

for some $u_{0} \in H^{1}\left(\mathbb{R}^{d}\right)$, some $t_{0} \in I$, and some $\varepsilon<\varepsilon_{0}$, then there exists a solution $u \in C\left(I ; H^{1}\left(\mathbb{R}^{d}\right)\right)$ to the defocusing energy-critical NLS:

$$
i \partial_{t} u+\Delta u=|u|^{\frac{4}{d-2}} u
$$

with $u\left(t_{0}\right)=u_{0}$ such that

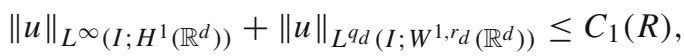

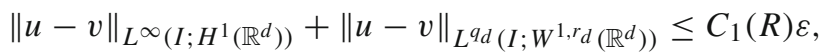

where $C_{1}(R)$ is a non-decreasing function of $R$.

With Lemmas 4.1 and 4.3 in hand, we can repeat the argument in Proposition 3.3 and obtain the following proposition. The proof is essentially identical to that of Proposition 3.3 and hence we omit details. We point out that, in applying the perturbation lemma (Lemma 4.3) with $e=\mathcal{N}_{1}(v+f)-\mathcal{N}_{1}(v)$, we use (32), which imposes the restriction $d \leq 6$.

Proposition 4.4 Let $3 \leq d \leq 6$. Given $T>0$, assume the following conditions ( $i$ )-(ii):

(i) $f \in L^{q_{d}}\left([0, T] ; W^{1, r_{d}}\left(\mathbb{R}^{d}\right)\right)$ and there exist $C, \theta>0$ such that

$$
\|f\|_{L^{q_{d}\left(I ; W^{1, r} d\left(\mathbb{R}^{d}\right)\right)}} \leq C|I|^{\theta}
$$

for any interval $I \subset[0, T]$.

(ii) Given a solution $v$ to (29), the following a priori $H^{1}$-bound holds:

$$
\|v\|_{L^{\infty}\left([0, T] ; H^{1}\left(\mathbb{R}^{d}\right)\right)} \leq R
$$

for some $R \geq 1$. 
Then, there exists $\tau=\tau(R, \theta)>0$ such that, given any $t_{0} \in[0, T)$, a unique solution $v$ to (29) with $k=1$ exists on $\left[t_{0}, t_{0}+\tau\right] \cap[0, T]$. In particular, the condition (ii) guarantees existence of a unique solution $v$ to the perturbed NLS (29) on the entire interval $[0, T]$.

\subsection{Proof of Theorem 1.1(ii)}

As in Sect. 3.2, Theorem 1.1(ii) follows from applying Proposition 4.4 to (29) with $f=\Psi$, once we verify the hypotheses (i) and (ii).

Fix $T>0$. As in Sect. 3.2, the hypothesis (i) in Proposition 4.4 can easily be verified from Hölder's inequality in time, Markov's inequality, and Lemma 2.2(ii), once we note that

$$
r_{d}=\frac{2 d^{2}}{d^{2}-2 d+4} \leq \frac{2 d}{d-2} .
$$

Furthermore, the following almost sure a priori bound follows from Lemma 2.4 and Markov's inequality:

$$
\sup _{0 \leq t \leq T}(M(u(t))+E(u(t))) \leq C\left(\omega, T, M\left(u_{0}\right), E\left(u_{0}\right),\|\phi\|_{H S\left(L^{2} ; H^{1}\right)}\right)<\infty
$$

for a solution $u$ to (1) with $p=1+\frac{4}{d-2}$. Then, from (34) and Lemma 2.2(i), we obtain

$$
\begin{aligned}
\sup _{0 \leq t \leq T}\|v(t)\|_{H^{1}} & \leq \sup _{0 \leq t \leq T}\|u(t)\|_{H^{1}}+\sup _{0 \leq t \leq T}\|\Psi(t)\|_{H^{1}} \\
& \leq C\left(\omega, T, M\left(u_{0}\right), E\left(u_{0}\right),\|\phi\|_{H S\left(L^{2} ; H^{1}\right)}\right)<\infty
\end{aligned}
$$

almost surely. This shows that the hypothesis (ii) in Proposition 4.4 holds almost surely for some almost surely finite $R=R(\omega) \geq 1$. This proves Theorem 1.1(ii).

Acknowledgements T.O. was supported by the European Research Council (Grant No. 637995 "ProbDynDispEq"). M.O. was supported by JSPS KAKENHI Grant No. JP16K17624. The authors would like to thank the anonymous referee for helpful comments.

Open Access This article is licensed under a Creative Commons Attribution 4.0 International License, which permits use, sharing, adaptation, distribution and reproduction in any medium or format, as long as you give appropriate credit to the original author(s) and the source, provide a link to the Creative Commons licence, and indicate if changes were made. The images or other third party material in this article are included in the article's Creative Commons licence, unless indicated otherwise in a credit line to the material. If material is not included in the article's Creative Commons licence and your intended use is not permitted by statutory regulation or exceeds the permitted use, you will need to obtain permission directly from the copyright holder. To view a copy of this licence, visit http://creativecommons.org/licenses/by/4.0/. 


\section{Appendix A: On the application of Ito's lemma}

In this appendix, we briefly discuss the derivation of the a priori bounds on the mass and the energy stated in Lemma 2.4. The argument essentially follows from that by de Bouard-Debussche [14] but we indicate certain required modifications.

\section{A.1. Mass-critical case}

We first consider the mass-critical case. Given $N \in \mathbb{N}$, let $P_{N}$ denote a smooth frequency projection onto $\{|\xi| \leq N\}$ and set $\phi_{N}:=P_{N} \circ \phi$. Then, consider the following truncated SNLS:

$$
\left\{\begin{array}{l}
i \partial_{t} u_{N}+\Delta u_{N}=\mathcal{N}_{0}\left(u_{N}\right)+\phi_{N} \xi \\
\left.u_{N}\right|_{t=0}=P_{N} u_{0}
\end{array}\right.
$$

where $\mathcal{N}_{0}$ is as in (8). Note that $P_{N} u_{0} \in H^{1}\left(\mathbb{R}^{d}\right)$ and $\phi_{N} \in H S\left(L^{2} ; H^{1}\right)$. Therefore, it follows from [14] that (A.1) is globally well-posed for each $N \in \mathbb{N}$. Furthermore, from Proposition 3.2 in [14], we have

$$
M\left(u_{N}(t)\right)=M\left(P_{N} u_{0}\right)+2 \operatorname{Im} \sum_{n \in \mathbb{N}} \int_{0}^{t} \int_{\mathbb{R}^{d}} \overline{u_{N}\left(t^{\prime}\right)} \phi_{N} e_{n} d x d \beta_{n}\left(t^{\prime}\right)+2 t\left\|\phi_{N}\right\|_{H S\left(L^{2} ; L^{2}\right)}^{2}
$$

for any $t \geq 0$ and, as a consequence of (A.2) and the Burkholder-Davis-Gundy inequality (see, for example, [21, Theorem 3.28 on p. 166]), the a priori bound (13) holds for each $u_{N}$, with the constant $C_{1}$, independent of $N \in \mathbb{N}$.

Given $T_{0}>0$, let $0<T<\min \left(T^{*}, T_{0}\right)$ be a given stopping time as in Lemma 2.4(i) and $u$ be the solution to (1) constructed in Lemma 2.3(i). We now show that the solution $u_{N}$ to the truncated SNLS (A.1) converges to $u$ almost surely. Then, the a priori bound (13) for $u$ follows from that for $u_{N}$ mentioned above and the convergence of $u_{N}$ to $u$.

In the following, we suppress the spatial domain $\mathbb{R}^{d}$ for simplicity of the presentation. Given $R>0$, define a stopping time $T_{1}$ by setting

$$
T_{1}=T_{1}(R):=\inf \left\{\tau \geq 0:\|u\|_{L_{t, x}^{\frac{2(d+2)}{d}}([0, \tau])} \geq R\right\}
$$

and set $T_{2}:=\min \left(T, T_{1}\right)$. In view of the blowup alternative in Lemma 2.3, we have

$$
\|u\| \underset{L_{t, x} \frac{2(d+2)}{d}([0, T])}{<\infty}
$$

almost surely and hence we conclude that $T_{2} \nearrow T$ almost surely as $R \rightarrow \infty$.

Given small $\eta>0$ (to be chosen later), we divide the interval $\left[0, T_{2}\right]$ into $J=$ $J(R, \eta)$ many random subintervals $I_{j}=I_{j}(\omega)=\left[t_{j}, t_{j+1}\right]$ with $t_{0}=0<t_{1}<\cdots<$ 
$t_{J-1}<t_{J}=T_{2}$ such that

$$
\|u\| \underset{L_{t, x} \frac{2(d+2)}{d}\left(I_{j}\right)}{\sim \eta} \sim \eta
$$

for $j=0,1, \ldots, J-1$.

Define the truncated stochastic convolution $\Psi_{N}$ by

$$
\Psi_{N}(t):=-i \sum_{n \in \mathbb{N}} \int_{0}^{t} S\left(t-t^{\prime}\right) \phi_{N} e_{n} d \beta_{n}\left(t^{\prime}\right)
$$

and set

$$
\begin{aligned}
C_{N}^{(j)}\left(\omega, u_{0}, \phi\right) & =\left\|u\left(t_{j}\right)-u_{N}\left(t_{j}\right)\right\|_{L^{2}} \\
& +\left\|\Psi-\Psi_{N}\right\|_{L_{t, x} \frac{2(d+2)}{d}}\left(\left[0, T_{2}\right]\right)
\end{aligned}+\left\|\Psi-\Psi_{N}\right\|_{L^{\infty}\left(\left[0, T_{2}\right] ; L^{2}\right)}
$$

for $j=0,1, \ldots, J-1$. Then, it follows from the Lebesgue dominated convergence theorem (applied to $\left.\left(\mathrm{Id}-P_{N}\right) u_{0}\right)$ and Lemma 2.2 that

$$
C_{N}^{(0)}\left(\omega, u_{0}, \phi\right) \longrightarrow 0
$$

almost surely as $N \rightarrow \infty$.

From the Strichartz estimates (Lemma 2.1), we have

$$
\begin{aligned}
& \left\|u-u_{N}\right\|_{L_{t, x}{ }^{\frac{2(d+2)}{d}}([0, \tau])} \lesssim\left\|u(0)-u_{N}(0)\right\|_{L^{2}} \\
& +\left(\|u\| \underset{L_{t, x}^{\frac{2(d+2)}{d}}([0, \tau])}{ }+\left\|u_{N}\right\| \underset{L_{t, x}^{\frac{2(d+2)}{d}}([0, \tau])}{ }\right)^{\frac{4}{d}}\left\|u-u_{N}\right\| \underset{L_{t, x}^{\frac{2(d+2)}{d}}([0, \tau])}{ } \\
& +\left\|\Psi-\Psi_{N}\right\| \underset{L_{t, x}^{\frac{2(d+2)}{d}}([0, \tau])}{ }
\end{aligned}
$$

for any subinterval $[0, \tau] \subset I_{0}=\left[0, t_{1}\right]$. Then, from (A.6) with (A.3) and (A.4), we obtain

$$
\begin{aligned}
\left\|u-u_{N}\right\|_{L_{t, x}^{\frac{2(d+2)}{d}}([0, \tau])} \lesssim & C_{N}^{(0)}\left(\omega, u_{0}, \phi\right) \\
& +\left(\eta+\left\|u_{N}\right\|_{L_{t, x}^{\frac{2(d+2)}{d}}([0, \tau])}\right)^{\frac{4}{d}}\left\|u-u_{N}\right\|_{L_{t, x}^{\frac{2(d+2)}{d}}}([0, \tau])
\end{aligned}
$$

for any $0 \leq \tau \leq t_{1}$. By taking $\eta>0$ sufficiently small, a standard continuity argument with (A.7) and (A.5) yields

$$
\left\|u_{N}\right\| \underset{L_{t, x}}{\frac{2(d+2)}{d}\left(I_{0}\right)} \sim \eta,
$$

uniformly in $N \geq N_{0}(\omega)$. 
Applying Lemma 2.1 once again with (A.3) and (A.8), we then have

$$
\begin{aligned}
& \left\|u-u_{N}\right\|_{L^{\infty}\left(I_{0} ; L^{2}\right)}+\left\|u-u_{N}\right\| \frac{2(d+2)}{L_{t, x}^{d}}\left(I_{0}\right) \\
& \lesssim\left\|u(0)-u_{N}(0)\right\|_{L^{2}}+\eta^{\frac{4}{d}}\left\|u-u_{N}\right\|{ }_{L_{t, x}}^{\frac{2(d+2)}{d^{d}}}\left(I_{0}\right) \\
& +\left\|\Psi-\Psi_{N}\right\|_{L^{\infty}\left(I_{0} ; L^{2}\right)}+\left\|\Psi-\Psi_{N}\right\| \underset{L_{t, x}{ }^{\frac{2(d+2)}{d}}\left(I_{0}\right)}{ }
\end{aligned}
$$

uniformly in $N \geq N_{0}(\omega)$. Thus, from (A.4) and (A.5), we conclude that

$$
\left\|u-u_{N}\right\|_{L^{\infty}\left(I_{0} ; L^{2}\right)} \lesssim C_{N}^{(0)}\left(\omega, u_{0}, \phi\right) \longrightarrow 0
$$

as $N \rightarrow \infty$. In particular, we have

$$
\left\|u\left(t_{1}\right)-u_{N}\left(t_{1}\right)\right\|_{L^{2}} \lesssim C_{N}^{(0)}\left(\omega, u_{0}, \phi\right) \longrightarrow 0
$$

as $N \rightarrow \infty$. By repeating the argument above, we have

$$
\left\|u_{N}\right\|_{L_{t, x}}^{\frac{2(d+2)}{d}\left(I_{1}\right)} \sim \eta
$$

uniformly in $N \geq N_{1}(\omega)$. Together with (A.9), this yields

$$
\left\|u-u_{N}\right\|_{L^{\infty}\left(I_{1} ; L^{2}\right)} \lesssim C_{N}^{(1)}\left(\omega, u_{0}, \phi\right) \longrightarrow 0
$$

as $N \rightarrow \infty$.

By successively applying the argument above to the interval $I_{j}, j=0,1, \ldots, J-1$, we conclude that

$$
\left\|u-u_{N}\right\|_{L^{\infty}\left(I_{j} ; L^{2}\right)} \lesssim C_{N}^{(j)}\left(\omega, u_{0}, \phi\right) \longrightarrow 0
$$

as $N \rightarrow \infty$. Therefore, recalling that $J=J(R, \eta)$ depends only on $R>0$ and an absolute constant $\eta>0$, we obtain

$$
\left\|u-u_{N}\right\|_{L^{\infty}\left(\left[0, T_{2}\right] ; L^{2}\right)} \lesssim \sum_{j=0}^{J-1} C_{N}^{(j)}\left(\omega, u_{0}, \phi\right) \longrightarrow 0 .
$$

By the almost sure convergence of $u_{N}$ to $u$ in $C\left(\left[0, T_{2}\right] ; L^{2}\left(\mathbb{R}^{d}\right)\right)$, Fatou's lemma, and the uniform bound (13) for $u_{N}$, we then have

$$
\begin{aligned}
\mathbb{E}\left[\sup _{0 \leq t \leq T_{2}} M(u(t))\right] & =\mathbb{E}\left[\lim _{N \rightarrow \infty} \sup _{0 \leq t \leq T_{2}} M\left(u_{N}(t)\right)\right] \\
& \leq \lim _{N \rightarrow \infty} \mathbb{E}\left[\sup _{0 \leq t \leq T_{2}} M\left(u_{N}(t)\right)\right] \leq C_{1} .
\end{aligned}
$$


Finally, from the almost sure convergence of $T_{2}=T_{2}(R)$ to $T$, as $R \rightarrow \infty$, and Fatou's lemma, we conclude the bound (13) for $u$. This proves Lemma 2.4(i).

\section{A.2. Energy-critical case}

Next, we consider the energy-critical case. In the following, we only discuss the a priori bound on the energy:

$$
\mathbb{E}\left[\sup _{0 \leq t \leq T} E(u(t))\right] \leq C_{2}
$$

since the a priori bound on the mass follows in a similar but simpler manner.

Lemma 4.5 Assume the hypotheses in Lemma 2.3(ii). Then, for any stopping time $T$ such that $0<T<T^{*}$ almost surely, we have

$$
\begin{aligned}
E(u(T))= & E\left(u_{0}\right)-\operatorname{Im} \sum_{n \in \mathbb{N}} \int_{0}^{T} \int_{\mathbb{R}^{d}} \overline{\left(\Delta u-\mathcal{N}_{1}(u)\right)\left(t^{\prime}\right)} \phi e_{n} d x d \beta_{n}\left(t^{\prime}\right) \\
& +\frac{d}{d-2} \sum_{n \in \mathbb{N}} \int_{0}^{T} \int_{\mathbb{R}^{d}}\left|u\left(t^{\prime}\right)\right|^{\frac{4}{d-2}}\left|\phi e_{n}\right|^{2} d x d t^{\prime}+T\|\phi\|_{H S\left(L^{2} ; \dot{H}^{1}\right)}^{2},
\end{aligned}
$$

where $u$ is the solution to the energy-critical SNLS (1) with $p=1+\frac{4}{d-2}, \mathcal{N}_{1}$ is as in (8), and $T^{*}$ is the forward maximal time of existence.

Once we prove Lemma 4.5, the bound (A.10) follows from the Burkholder-DavisGundy inequality.

Proof A direct calculation shows that

$$
\begin{aligned}
E^{\prime}(u(t))(v)= & \operatorname{Re} \int_{\mathbb{R}^{d}}\left(\nabla u(t) \cdot \overline{\nabla v}+\mathcal{N}_{1}(u)(t) \bar{v}\right) d x, \\
E^{\prime \prime}(u(t))\left(v_{1}, v_{2}\right)= & \operatorname{Re} \int_{\mathbb{R}^{d}} \nabla v_{1} \cdot \overline{\nabla v_{2}} d x+\operatorname{Re} \int_{\mathbb{R}^{d}}|u(t)|^{\frac{4}{d-2}} v_{1} \overline{v_{2}} d x \\
& +\frac{4}{d-2} \int_{\mathbb{R}^{d}}|u(t)|^{\frac{4}{d-2}}-2 \operatorname{Re}\left(u(t) \overline{v_{1}}\right) \operatorname{Re}\left(u(t) \overline{v_{2}}\right) d x
\end{aligned}
$$

for $v, v_{1}, v_{2} \in H^{1}\left(\mathbb{R}^{d}\right)$. Thus, a formal application of Ito's lemma to $E(u(t))$ yields (A.11). It remains to justify the application of Ito's lemma.

As in the proof of Proposition 3.3 in [14], given $N \in \mathbb{N}$, we consider the following truncated problem:

$$
\left\{\begin{array}{l}
i \partial_{t} u_{N}+\Delta u_{N}=P_{N} \mathcal{N}_{1}\left(u_{N}\right)+\phi_{N} \xi \\
\left.u_{N}\right|_{t=0}=P_{N} u_{0},
\end{array} \quad(t, x) \in \mathbb{R}_{+} \times \mathbb{R}^{d},\right.
$$


where $P_{N}$ and $\phi_{N}$ are the same as those in Sect. 1. Since the frequency truncation is harmless, the same well-posedness result as in Lemma 2.3 (ii) holds for the truncated SNLS (A.12). Moreover, by considering the corresponding Duhamel formulation for (A.12), we have $u_{N}=P_{3 N} u_{N}$. We can therefore apply Ito's lemma (see Theorem 4.32 in [12]) to $E\left(u_{N}(t)\right)$ and obtain

$$
\begin{aligned}
E\left(u_{N}(t)\right)= & E\left(P_{N} u_{0}\right)-\operatorname{Im} \sum_{n \in \mathbb{N}} \int_{0}^{t} \int_{\mathbb{R}^{d}} \overline{\left(\Delta u_{N}-\mathcal{N}_{1}\left(u_{N}\right)\right)\left(t^{\prime}\right)} \phi_{N} e_{n} d x d \beta_{n}\left(t^{\prime}\right) \\
& +\operatorname{Im} \int_{0}^{t} \int_{\mathbb{R}^{d}} \overline{\Delta u_{N}\left(t^{\prime}\right)}\left(\operatorname{Id}-P_{N}\right) \mathcal{N}_{1}\left(u_{N}\right)\left(t^{\prime}\right) d x d t^{\prime} \\
& +\frac{d}{d-2} \sum_{n \in \mathbb{N}} \int_{0}^{t} \int_{\mathbb{R}^{d}}\left|u_{N}\left(t^{\prime}\right)\right|^{\frac{4}{d-2}}\left|\phi_{N} e_{n}\right|^{2} d x d t^{\prime}+t\left\|\phi_{N}\right\|_{H S\left(L^{2} ; \dot{H}^{1}\right)}^{2}
\end{aligned}
$$

for $0<t<T_{N}^{*}$, where $T_{N}^{*}$ is the forward maximal time of existence for the solution $u_{N}$ to $(\mathrm{A} .12)$.

Given $R>0$, define a stopping $T_{1}$ by setting

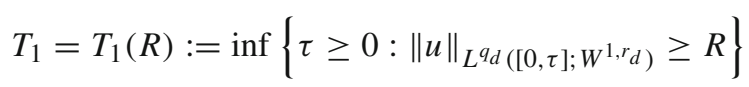

and set $T_{2}:=\min \left(T, T_{1}\right)$, where $T$ is the stopping time given in Lemma 2.4(ii) with $0<T<\min \left(T^{*}, T_{0}\right)$. In view of the blowup alternative in Lemma 2.3, we have

$$
\|u\|_{L^{q_{d}\left([0, T] ; W^{1, r} d\right)}}<\infty
$$

almost surely and hence we conclude that $T_{2} \nearrow T$ almost surely as $R \rightarrow \infty$.

From (30) and (32), we have

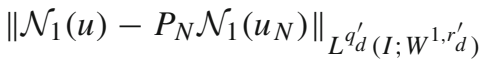

$$
\begin{aligned}
& \lesssim\left\|\left(\operatorname{Id}-P_{N}\right) \mathcal{N}_{1}(u)\right\|_{L^{q_{d}^{\prime}\left(I ; W^{\left.1, r_{d}^{\prime}\right)}\right.}}+\left\|\mathcal{N}_{1}(u)-\mathcal{N}_{1}\left(u_{N}\right)\right\|_{L_{d\left(I ; W^{1, r_{d}^{\prime}}\right.}^{q^{\prime}}}
\end{aligned}
$$

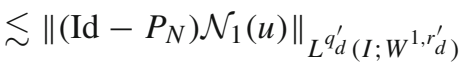

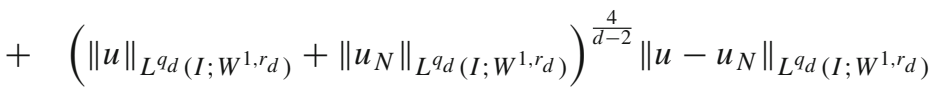

for any interval $I \subset\left[0, T_{2}\right]$. It follows from the Lebesgue dominated convergence theorem and (A.14) that the first term on the right-hand side of (A.15) converges to 0 almost surely as $N \rightarrow \infty$. Accordingly, proceeding as in Sect. 1 , we conclude that $u_{N}$ converges to $u$ in $C\left(\left[0, T_{2}\right] ; H^{1}\left(\mathbb{R}^{d}\right)\right) \cap L^{q_{d}}\left(\left[0, T_{2}\right] ; W^{1, r_{d}}\left(\mathbb{R}^{d}\right)\right)$ almost surely. In particular, there exists an almost surely finite number $N_{0}(\omega) \in \mathbb{N}$ such that $T_{N}^{*} \geq T_{2}$ for any $N \geq N_{0}(\omega)$ and, as a result, (A.13) holds for any $0<t<T_{2}$ and $N \geq N_{0}(\omega)$. Moreover, from the definition of $T_{2}=T_{2}(R)$, we may assume

$$
\left\|u_{N}\right\|_{L^{q_{d}\left(\left[0, T_{2}\right] ; W^{1, r} d\right)}} \leq R+1
$$


for any $N \geq N_{0}(\omega)$.

This allows us to conclude that the third term on the right-hand side of (A.13) tends to 0 almost surely as $N \rightarrow \infty$. Indeed, by (30), (32), (A.14), (A.16), and the almost sure convergence of $u_{N}$ to $u$ in $L^{q_{d}}\left(\left[0, T_{2}\right] ; W^{1, r_{d}}\left(\mathbb{R}^{d}\right)\right)$, we have, for any $0 \leq t \leq T_{2}$,

$$
\begin{aligned}
& \left|\int_{0}^{t} \int_{\mathbb{R}^{d}} \overline{\Delta u_{N}\left(t^{\prime}\right)}\left(\mathrm{Id}-P_{N}\right) \mathcal{N}_{1}\left(u_{N}\right)\left(t^{\prime}\right) d x d t^{\prime}\right| \\
& \leq\left|\int_{0}^{t} \int_{\mathbb{R}^{d}}\left(\mathrm{Id}-P_{N}\right) \overline{\Delta u\left(t^{\prime}\right)} \cdot \mathcal{N}_{1}(u)\left(t^{\prime}\right) d x d t^{\prime}\right| \\
& +\left|\int_{0}^{t} \int_{\mathbb{R}^{d}} \overline{\left(\Delta u-\Delta u_{N}\right)\left(t^{\prime}\right)}\left(\mathrm{Id}-P_{N}\right) \mathcal{N}_{1}(u)\left(t^{\prime}\right) d x d t^{\prime}\right| \\
& +\left|\int_{0}^{t} \int_{\mathbb{R}^{d}} \overline{\Delta u_{N}\left(t^{\prime}\right)}\left(\mathrm{Id}-P_{N}\right)\left(\mathcal{N}_{1}(u)-\mathcal{N}_{1}\left(u_{N}\right)\right)\left(t^{\prime}\right) d x d t^{\prime}\right|
\end{aligned}
$$

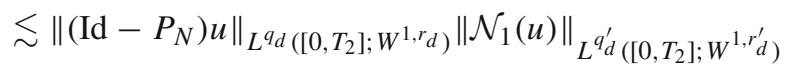

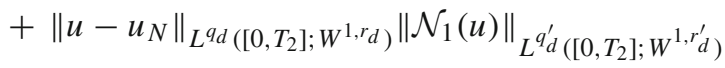

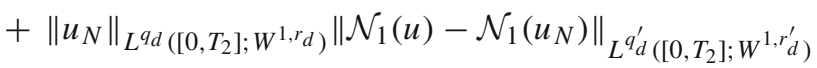

$$
\begin{aligned}
& \lesssim\left\|\left(\operatorname{Id}-P_{N}\right) u\right\|_{L^{q_{d}}\left(\left[0, T_{2}\right] ; W^{\left.1, r_{d}\right)}\right.}\|u\|_{L^{q_{d}\left(\left[0, T_{2}\right] ; W^{1, r_{d}}\right)}}^{\frac{d+2}{d-2}}
\end{aligned}
$$

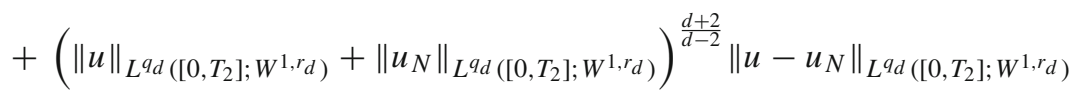

$$
\begin{aligned}
& \longrightarrow 0
\end{aligned}
$$

almost surely, as $N \rightarrow \infty$.

Let us now consider the second and fourth terms on the right-hand side of (A.13). As for the second term, we first consider the contribution from $\overline{\mathcal{N}_{1}\left(u_{N}\right)} \phi_{N} e_{n}$. By Hölder's inequality with (8) and Sobolev's embedding: $\dot{H}^{1}\left(\mathbb{R}^{d}\right) \hookrightarrow L^{\frac{2 d}{d-2}}\left(\mathbb{R}^{d}\right)$, we have

$$
\left|\int_{\mathbb{R}^{d}} \overline{\mathcal{N}_{1}\left(u_{N}\right)} \phi_{N} e_{n} d x\right| \leq\left\|u_{N}\right\|_{L^{\frac{2 d}{d-2}}}^{\frac{d+2}{d-2}}\left\|\phi_{N} e_{n}\right\|_{L^{\frac{2 d}{d-2}}} \lesssim\left\|u_{N}\right\|_{\dot{H}^{1}}^{\frac{d+2}{d-2}}\left\|\phi_{N} e_{n}\right\|_{\dot{H}^{1}} .
$$

Then, by Ito's isometry along with the independence of $\left\{\beta_{n}\right\}_{n \in \mathbb{N}}$, we obtain

$$
\begin{gathered}
\mathbb{E}\left[\mid \sum_{n \in \mathbb{N}} \int_{0}^{t} \int_{\mathbb{R}^{d}} \frac{\left.\left.\overline{\mathcal{N}_{1}\left(u_{N}\right)\left(t^{\prime}\right)} \phi_{N} e_{n} d x d \beta_{n}\left(t^{\prime}\right)\right|^{2}\right] \lesssim t \sum_{n \in \mathbb{N}}\left\|u_{N}\right\|_{L^{\infty}\left([0, t] ; \dot{H}^{1}\right)}^{\frac{2(d+2)}{d-2}}\left\|\phi_{N} e_{n}\right\|_{\dot{H}^{1}}^{2}}{\lesssim t\left\|u_{N}\right\|_{L^{\infty}\left([0, t] ; \dot{H}^{1}\right)}^{\frac{2(d+2)}{d-2}}\left\|\phi_{N}\right\|_{H S\left(L^{2} ; \dot{H}^{1}\right)}^{2}}\right.
\end{gathered}
$$


By integration by parts (in $x$ ) and Ito's isometry, we bound the contribution from $\overline{\Delta u_{N}} \phi_{N} e_{n}$ by

$$
\mathbb{E}\left[\left|\sum_{n \in \mathbb{N}} \int_{0}^{t} \int_{\mathbb{R}^{d}} \overline{\Delta u_{N}\left(t^{\prime}\right)} \phi_{N} e_{n} d x d \beta_{n}\left(t^{\prime}\right)\right|^{2}\right] \lesssim t\left\|u_{N}\right\|_{L^{\infty}\left([0, t] ; \dot{H}^{1}\right)}^{2}\left\|\phi_{N}\right\|_{H S\left(L^{2} ; \dot{H}^{1}\right)}^{2} .
$$

As for the fourth term on the right-hand side of (A.13), it follows from Hölder's and Sobolev's inequalities that

$$
\begin{aligned}
\sum_{n \in \mathbb{N}} \int_{\mathbb{R}^{d}}\left|u_{N}\right|^{\frac{4}{d-2}}\left|\phi_{N} e_{n}\right|^{2} d x & \leq\left\|u_{N}\right\|_{L^{\frac{2 d}{d-2}}}^{\frac{4}{d-2}} \sum_{n \in \mathbb{N}}\left\|\phi_{N} e_{n}\right\|_{L^{\frac{2 d}{d-2}}}^{2} \\
& \lesssim\left\|u_{N}\right\|_{\dot{H}^{1}}^{\frac{4}{d-2}}\left\|\phi_{N}\right\|_{H S\left(L^{2} ; \dot{H}^{1}\right)^{\cdot}}^{2}
\end{aligned}
$$

Since $3 \leq d \leq 6$, we have $\frac{4}{d-2} \geq 1$, which implies that difference estimates on the contributions from $u_{N}$ and $u$ for (A.18), (A.19), and (A.20) also hold. Therefore, by in view of (A.17) and (the difference estimates for) (A.18), (A.19), and (A.20), we obtain (A.11) by taking $N \rightarrow \infty$ in (A.13) and then $R \rightarrow \infty$. This concludes the proof of Lemma 4.5.

\section{References}

1. Barbu, V., Röckner, M., Zhang, D.: Stochastic nonlinear Schrödinger equations with linear multiplicative noise: rescaling approach. J. Nonlinear Sci. 24(3), 383-409 (2014)

2. Barbu, V., Röckner, M., Zhang, D.: Stochastic nonlinear Schrödinger equations. Nonlinear Anal. 136, 168-194 (2016)

3. Barbu, V., Röckner, M., Zhang, D.: Stochastic nonlinear Schrödinger equations: no blow-up in the non-conservative case. J. Differ. Equ. 263(11), 7919-7940 (2017)

4. Bényi, Á., Oh, T., Pocovnicu, O.: On the probabilistic Cauchy theory of the cubic nonlinear Schrödinger equation on $\mathbb{R}^{d}, d \geq 3$. Trans. Am. Math. Soc. Ser. B 2, 1-50 (2015)

5. Bourgain, J.: Global wellposedness of defocusing critical nonlinear Schrödinger equation in the radial case. J. Am. Math. Soc. 12(1), 145-171 (1999)

6. Cazenave, T., Weissler, F.: Some remarks on the nonlinear Schrödinger equation in the critical case. In: Nonlinear Semigroups, Partial Differential Equations and Attractors (Washington, DC, 1987). Lecture Notes in Mathematics, vol. 1394, pp. 18-29

7. Cheung, K., Li, G., Oh, T.: Almost conservation laws for stochastic nonlinear Schrödinger equations. arXiv arXiv:1910.14558 [math.AP]

8. Cheung, K., Pocovnicu, O.: On the local well-posedness of the stochastic cubic nonlinear Schrödinger equation on $\mathbb{R}^{d}, d \geq 3$, with supercritical noise (preprint)

9. Christ, M., Colliander, J., Tao, T.: Ill-posedness for nonlinear Schrödinger and wave equations. arXiv:math/0311048 [math.AP]

10. Colliander, J., Keel, M., Staffilani, G., Takaoka, H., Tao, T.: Almost conservation laws and global rough solutions to a nonlinear Schrödinger equation. Math. Res. Lett. 9(5-6), 659-682 (2002)

11. Colliander, J., Keel, M., Staffilani, G., Takaoka, H., Tao, T.: Global well-posedness and scattering for the energy-critical nonlinear Schrödinger equation in $\mathbb{R}^{3}$. Ann. Math. 167(3), 767-865 (2008)

12. Da Prato, G., Zabczyk, J.: Stochastic Equations in Infinite Dimensions. Encyclopedia of Mathematics and Its Applications, vol. 152, 2nd edn. Cambridge University Press, Cambridge (2014)

13. de Bouard, A., Debussche, A.: On the effect of a noise on the solutions of the focusing supercritical nonlinear Schrödinger equation. Probab. Theory Relat. Fields 123(1), 76-96 (2002) 
14. de Bouard, A., Debussche, A.: The stochastic nonlinear Schrödinger equation in $H^{1}$. Stoch. Anal. Appl. 21(1), 97-126 (2003)

15. Dodson, B.: Global well-posedness and scattering for the defocusing, $L^{2}$-critical nonlinear Schrödinger equation when $d \geq 3$. J. Am. Math. Soc. 25(2), 429-463 (2012)

16. Dodson, B.: Global well-posedness and scattering for the defocusing, $L^{2}$-critical, nonlinear Schrödinger equation when $d=1$. Am. J. Math. 138(2), 531-569 (2016)

17. Dodson, B.: Global well-posedness and scattering for the defocusing, $L^{2}$-critical, nonlinear Schrödinger equation when $d=2$. Duke Math. J. 165(18), 3435-3516 (2016)

18. Fan, C., $\mathrm{Xu}, \mathrm{W} .:$ Global well-posedness for the defocusing mass-critical stochastic nonlinear Schrödinger equation on $\mathbb{R}$ at $L^{2}$ regularity. arXiv:1810.07925 [math.AP]

19. Ginibre, J., Velo, G.: On a class of nonlinear Schrödinger equations. I. The Cauchy problem, general case. J. Funct. Anal. 32(1), 1-32 (1979)

20. Ginibre, J., Velo, G.: Smoothing properties and retarded estimates for some dispersive evolution equations. Commun. Math. Phys. 144(1), 163-188 (1992)

21. Karatzas, I., Shreve, S.: Brownian Motion and Stochastic Calculus. Graduate Texts in Mathematics, vol. 113, 2nd edn. Springer, New York (1991)

22. Kato, T.: On nonlinear Schrödinger equations. Ann. Inst. Henri. Poincaré Phys. Théor. 46(1), 113-129 (1987)

23. Keel, M., Tao, T.: Endpoint Strichartz estimates. Am. J. Math. 120(5), 955-980 (1998)

24. Killip, R., Oh, T., Pocovnicu, O., Vişan, M.: Global well-posedness of the Gross-Pitaevskii and cubicquintic nonlinear Schrödinger equations with non-vanishing boundary conditions. Math. Res. Lett. 19(5), 969-986 (2012)

25. Killip, R., Vişan, M.: Nonlinear Schrödinger equations at critical regularity. Evolution Equations. Clay Mathematics Proceedings, vol. 17, pp. 325-437. American Mathematical Society, Providence (2013)

26. Kishimoto, N.: A remark on norm inflation for nonlinear Schrödinger equations. Commun. Pure Appl. Anal. 18(3), 1375-1402 (2019)

27. Kuo, H.S.: Introduction to Stochastic Integration (Universitext). Springer, New York (2006)

28. Oh, T.: A remark on norm inflation with general initial data for the cubic nonlinear Schrödinger equations in negative Sobolev spaces. Funkc. Ekvac. 60, 259-277 (2017)

29. Oh, T., Okamoto, M., Pocovnicu, O.: On the probabilistic well-posedness of the nonlinear Schrödinger equations with non-algebraic nonlinearities. Discret. Contin. Dyn. Syst. A. 39(6), 3479-3520 (2019)

30. Oh, T., Pocovnicu, O., Wang, Y.: On the stochastic nonlinear Schrödinger equations with non-smooth additive noise. Kyoto J. Math. (to appear)

31. Ryckman, E., Visan, M.: Global well-posedness and scattering for the defocusing energy-critical nonlinear Schrödinger equation in $\mathbb{R}^{1+4}$. Am. J. Math. 129(1), 1-60 (2007)

32. Strichartz, R.S.: Restrictions of Fourier transforms to quadratic surfaces and decay of solutions of wave equations. Duke Math. J. 44(3), 705-714 (1977)

33. Tao, T., Vişan, M.: Stability of energy-critical nonlinear Schrödinger equations in high dimensions. Electron. J. Differ. Equ. 2005, 1-28 (2005)

34. Tao, T., Vişan, M., Zhang, X.: Global well-posedness and scattering for the defocusing mass-critical nonlinear Schrödinger equation for radial data in high dimensions. Duke Math. J. 140(1), 165-202 (2007)

35. Tao, T., Vişan, M., Zhang, X.: The nonlinear Schrödinger equation with combined power-type nonlinearities. Commun. Partial Differ. Equ. 32(7-9), 1281-1343 (2007)

36. Tsutsumi, Y.: $L^{2}$-solutions for nonlinear Schrödinger equations and nonlinear groups. Funkcial. Ekvac. 30(1), 115-125 (1987)

37. Vişan, M.: The defocusing energy-critical nonlinear Schrödinger equation in higher dimensions. Duke Math. J. 138(2), 281-374 (2007)

38. Yajima, K.: Existence of solutions for Schrödinger evolution equations. Commun. Math. Phys. 110(3), 415-426 (1987)

39. Zhang, D.: Stochastic nonlinear Schrödinger equations in the defocusing mass and energy critical cases. arXiv:1811.00167 [math.PR]

Publisher's Note Springer Nature remains neutral with regard to jurisdictional claims in published maps and institutional affiliations. 